PoS $\quad \begin{aligned} & \text { PROCEEDINGS } \\ & \text { OF SCIENCE }\end{aligned}$

\title{
Field theories invariant under one-parameter fermionic symmetry
}

\author{
O. Radchenko* and P. Lavrov \\ Tomsk State Pedagogical University, Russia \\ E-mail: radchenkodt.spu.edu.ru, lavrovatspu.edu.ru
}

We research the quantum properties of field theories invariant under an one-parameter nilpotent fermion symmetry.

Proceedings of the Corfu Summer Institute 2014 "School and Workshops on Elementary Particle Physics and Gravity",

3-21 September 2014

Corfu, Greece

* Speaker. 


\section{Introduction}

There are many examples of field models with quantum action invariant under one-parameter fermion symmetry which appear in several ways within modern quantum field theory.

The well-known example is the Faddeev -Popov action for Yang -Mills fields [1]

$$
S_{\mathrm{FP}}(\phi)=S_{Y M}+S_{g f}+S_{g h}
$$

where

$$
\phi^{A}=\left(A^{\mu a}, B^{a}, C^{a}, \bar{C}^{a}\right)
$$

is the set of dynamical fields in the Lagrangian formalism of the Yang-Mills theory which contains vector fields $A^{\mu a}$, ghost $C^{a}$ and antighost $\bar{C}^{a}$, auxiliary fields $B^{a}$ introducing the gauge; $S_{Y M}$ is the Yang-Mills action, the second and the third terms have form

$$
\begin{aligned}
S_{g h} & =\bar{C}^{a} \partial_{\mu} D^{\mu a b} C^{b}, \\
D_{\mu}^{a b} & =\delta^{a b} \partial_{\mu}+f^{a c b} A_{\mu}^{c}, \\
S_{g f} & =B^{a} \partial_{\mu} A^{\mu a} .
\end{aligned}
$$

This action is invariant under remarkable nilpotent transformations known as BRST - transformations $[2,3]$

$$
\begin{gathered}
\delta_{\mathrm{B}} S_{\mathrm{FP}}(\phi)=0, \\
\delta_{\mathrm{B}} A_{\mu}^{a}=D_{\mu}^{a b}(A) C^{b} \lambda, \quad \delta_{\mathrm{B}} C^{a}=\frac{1}{2} f^{a b c} C^{b} C^{c} \lambda, \\
\delta_{\mathrm{B}} \bar{C}^{a}=B^{a} \lambda,
\end{gathered}
$$

where $\lambda$ is a constant Grassmann parameter $(\varepsilon(\lambda)=1), \lambda^{2}=0$.

The next example is the well-known Curci-Ferrari model of non -Abelian massive vector fields possesses fermion invariance connected with the modified BRST and modified anti-BRST transformations [4].

The Curci-Ferrari model is described by the action

$$
S=S_{Y M}+S_{g f}+S_{m},
$$

where $S_{Y M}$ is the Yang-Mills action, the second term in (1.7) has form

$$
\begin{gathered}
S_{g f}=B^{a} \partial^{\mu} A_{\mu}^{a}+\bar{C}^{a} \partial^{\mu} D_{\mu}^{a b} C^{b}+\frac{\beta}{2} B^{a} B^{a}+\frac{\beta}{4} N^{a} N^{a}-\frac{\beta}{2} B^{a} N^{a}, \\
N^{a}=N^{a}(C, \bar{C})=f^{a b c} \bar{C}^{b} C^{a},
\end{gathered}
$$

$\beta$ is a parameter of the model, $f^{a b c}$ are structure constants of Lie group.

The action $S_{m}$

$$
S_{m}=\frac{1}{2} m^{2} A_{\mu}^{a} A^{a \mu}+\beta m^{2} \bar{C}^{a} C^{a}
$$


contains a mass $\mathrm{m}$ for the vector fields $A_{\mu}^{a}$.

The action of the Curci-Ferrari model is not invariant under BRST transformation

$$
\delta_{B} S \neq 0
$$

though it is invariant $\delta_{m B} S=0$ under the modified BRST transformation

$$
\begin{array}{rlrl}
\delta_{m B} A_{\mu}^{a} & =D_{\mu}^{a b} C^{b} \theta, & \delta_{m B} C^{a}=\frac{1}{2} f^{a b c} C^{b} C^{c} \theta \\
\delta_{m B} \bar{C}^{a} & =B^{a} \theta, & \delta_{m B} B^{a} & =m^{2} C^{a} \theta
\end{array}
$$

where $\theta$ is a constant Grassman parameter.

In contrast to the BRST (or anti-BRST) transformation the modified BRST (or modified antiBRST) transformation is not nilpotent $\delta_{m B}^{2} S \neq 0$.

The next example is the action appearing in generalized Hamiltonian formalism proposed by I.A. Batalin, E.S. Fradkin, G.A. Vilkovisky [5, 6]

$$
W_{\psi}=\int\left[\left(\frac{1}{2}\right) z^{i}(t) \omega_{i k} \dot{z}^{k}(t)-H(t)\right]
$$

where $z^{i}=(q ; p)$ is a complete set of canonical variables specific to the extended phase space of generalized Hamiltonian formalism,

$$
\dot{z}^{k}(t)=\frac{d z^{k}(t)}{d t}
$$

$\omega_{i k}$ is inverse metric to Poisson structure

$$
\left\{z^{i}, z^{k}\right\}=\omega^{i k}=\text { const } .
$$

$H(t)$ is full Hamiltonian of the system: $H(t)=\mathscr{H}+\{\Omega, \psi\}, \mathscr{H}=H_{0}+\ldots$ is Hamiltonian of the system, $H_{0}$ - initial classical Hamiltonian for the given dynamical system with constraints.

This method uses two fundamental objects called BRST charge $\Omega$ and generalized Hamiltonian $\mathrm{H}$ which are solutions to these equations formulated in terms of super Poisson brackets:

$$
\{\Omega, \Omega\}=0, \quad\{\Omega, \mathscr{H}\}=0,
$$

$\Omega=c T+\ldots$ is BRST charge, $\mathrm{c}$ are ghost variables, $\mathrm{T}$ is a set of first - class constraints.

The action is invariant under the transformation

$$
\bar{z}^{k}(t)=\left.\bar{z}^{k}\right|_{z \rightarrow z(t)}=z^{k}(t)+\left\{z^{k}, \Omega\right\}_{t} \mu
$$

where $\mu$ is a constant Grassmann parameter, $\mu^{2}=0$.

In recent years there is also an interest to such theores. For example, superextension of sigma models [7] leads to actions again invariant under fermion transformations.

The latest attempts to formulate Yang-Mills fields in a form being free of the Gribov problem give another examples of actions invariant under some nilpotent fermion transformations [8].

In 2013 a new realization of supersymmetry, called scalar supersymmetry, has been proposed [9]. 


\section{One-parameter fermion symmetry}

Our starting point is a theory of variables $\phi=\left\{\phi^{i}\right\}$ with Grassmann parities $\varepsilon\left(\phi^{i}\right)=\varepsilon_{i}$. We assume a non-degenerate action $S(\phi)$ of the theory so that the generating functional of Green functions is given by the standard functional integral

$$
Z(J)=\int \mathscr{D} \phi \exp \left\{\frac{i}{\hbar}[S(\phi)+J \phi]\right\},
$$

here $J \phi=J_{i} \phi^{i}, \varepsilon\left(J_{i}\right)=\varepsilon_{i}$.

We suppose invariance of $S(\phi)$ under fermion transformations

$$
\phi^{i} \mapsto \phi^{i}=\varphi^{i}\left(\phi^{\prime}\right), \quad \varphi^{i}(\phi)=\phi^{i}+R^{i}(\phi) \xi, \quad \xi^{2}=0,
$$

so that

$$
S_{, i}(\phi) R^{i}(\phi)=0
$$

Here $\xi$ is an odd Grassmann parameter and $R^{i}(\phi)$ are generators of fermion transformations with $\varepsilon\left(R^{i}\right)=\varepsilon_{i}+1$.

Consider some consequences of the invariance on quantum level. Making the change of variables in the functional integral we have

$$
Z(J)=\int \mathscr{D} \phi \operatorname{sDet} M(\phi) \exp \left\{\frac{i}{\hbar}[S(\varphi(\phi))+J \varphi(\phi)]\right\}
$$

where sDet $M$ means the superdeterminant of supermatrix $M$ with matrix elements

$$
M_{j}^{i}(\phi)=\delta_{j}^{i}+(-1)^{\varepsilon_{j}} \frac{\delta R^{i}(\phi)}{\delta \phi^{j}} \xi, \quad \varepsilon\left(M_{j}^{i}\right)=\varepsilon_{i}+\varepsilon_{j} .
$$

In general, for the theory under consideration this superdeterminant is not equal to unity

$$
\begin{aligned}
\operatorname{sDet} M(\phi) & =\exp \{\operatorname{sTr} \ln M(\phi)\}=\exp \left\{\frac{\delta R^{i}(\phi)}{\delta \phi^{i}} \xi\right\}= \\
& =1+\frac{\delta R^{i}(\phi)}{\delta \phi^{i}} \xi=1+R_{, i}^{i}(\phi) \xi
\end{aligned}
$$

It leads to the following presentation of functional $Z(J)$

$$
Z(J)=\int \mathscr{D} \phi\left(1+R_{, i}^{i}(\phi) \xi+\frac{i}{\hbar} J_{i} R^{i}(\phi) \xi\right) \exp \left\{\frac{i}{\hbar}[S(\phi)+J \phi]\right\}
$$

from which the identity follows

$$
\int \mathscr{D} \phi\left(R_{, i}^{i}(\phi)+\frac{i}{\hbar} J_{i} R^{i}(\phi)\right) \exp \left\{\frac{i}{\hbar}[S(\phi)+J \phi]\right\}=0 .
$$

With the help of usual manipulations this identity can be written in closed form with respect to $Z(J)$

$$
\left[J_{i} R^{i}\left(\frac{\hbar}{i} \frac{\delta}{\delta J}\right)-i \hbar R_{, i}^{i}\left(\frac{\hbar}{i} \frac{\delta}{\delta J}\right)\right] Z(J)=0
$$


This identity is nothing but the Ward identity for generating functional of Green functions. The existence of this identity is direct consequence of invariance under fermion transformation of $S(\phi)$.

To simplify presentation of the Ward identity we define the extended generating functional of Green functions by introducing additional sources $K_{i}$ with Grassmann parities opposite to fields $\phi^{i}$, $\varepsilon\left(K_{i}\right)=\varepsilon_{i}+1$

$$
Z(J, K)=\int \mathscr{D} \phi \exp \left\{\frac{i}{\hbar}[S(\phi, K)+J \phi]\right\}
$$

where

$$
S(\phi, K)=S(\phi)+K_{i} R^{i}(\phi)
$$

In general, the action $S(\phi, K)$ is not invariant under fermion transformation

$$
\hat{s} S(\phi, K)=K_{i} \hat{s} R^{i}(\phi) \neq 0
$$

where the operator $\hat{s}$ of fermion transformation was used. Action of this operator on arbitrary functional $X$ is given by

$$
\hat{s} X=\frac{\delta X}{\delta \phi^{i}} R^{i}=X_{, i} R^{i}
$$

There is an evident relation

$$
\left.Z(J, K)\right|_{K=0}=Z(J)
$$

In terms of $Z(J, K)$ the Ward identity reads

$$
J_{i} \frac{\delta Z(J, K)}{\delta K_{i}}=i \hbar R_{, i}^{i}\left(\frac{\hbar}{i} \frac{\delta}{\delta J}\right) Z(J, K)
$$

Note that the left side of the Ward identity has the local form while the right side is nonlocal.

\section{Field-dependent nilpotent fermion transformations}

Let us study more general type of fermion transformations when the parameter $\xi$ is replaced by a field-dependent functional $\xi(\phi)$

$$
\varphi^{i}(\phi)=\phi^{i}+R^{i}(\phi) \xi(\phi), \quad \xi^{2}(\phi)=0 .
$$

We will referee to these transformations as field-dependent fermion transformations. Note that the action $S=S(\phi)$ remains invariant under these transformations due to nilpotency of $\xi(\phi)$

$$
S(\phi)=S\left(\varphi\left(\phi^{\prime}\right)\right)=S\left(\phi^{\prime}\right)
$$

Using the technique described in [10] it is not difficult to find the explicit form of the superdeterminant of supermatrix

$$
M_{j}^{i}(\phi)=\delta_{j}^{i}+R^{i}(\phi) \xi_{, j}(\phi)+(-1)^{\varepsilon_{i}} R_{, j}^{i}(\phi) \xi(\phi),
$$


with the result

$$
\operatorname{sDet} M(\phi)=(1+\hat{s} \xi(\phi))^{-1}\left[1+R_{, i}^{i}(\phi) \xi(\phi)-\frac{\left(\hat{s}^{2} \xi(\phi)\right) \xi(\phi)}{1+\hat{s} \xi(\phi)}\right] .
$$

Here we took into account that the action of the square operator $\hat{s}$ on an arbitrary functional $X$ is given by

$$
\hat{s}^{2} X=\frac{\delta X}{\delta \phi^{i}} \frac{\delta R^{i}}{\delta \phi^{j}} R^{j}=X_{, i} R_{, j}^{i} R^{j}
$$

In what follows we restrict ourselves to the case when the operator $\hat{s}$ is nilpotent, $\hat{s}^{2}=0$

$$
\hat{s}^{2}=0 \quad \rightarrow \quad \frac{\delta R^{i}}{\delta \phi^{j}} R^{j}=0 .
$$

In particular, it means

$$
\hat{s} R^{i}=0
$$

and we find that the action $S(\phi, K)$ is invariant under field-dependent nilpotent fermion transformations

$$
S(\phi, K),{ }_{i} R^{i}(\phi)=0 .
$$

The invariance of $S(\phi, K)$ can be expressed in an unique form

$$
\frac{\delta S(\phi, K)}{\delta \phi^{i}} \frac{\delta S(\phi, K)}{\delta K_{i}}=0 .
$$

This equation is nothing but the Zinn-Justin equation appearing for the first time in quantization of non-abelian gauge fields [11].

Performing the change of variables in form of field-dependent nilpotent fermion transformations and using the explicit form of the Jacobian we have

$$
\begin{aligned}
\operatorname{sDet} M(\phi) & =\exp \left\{R_{, i}^{i}(\phi) \xi(\phi)-\ln (1+\hat{s} \xi(\phi))\right\}= \\
& =(1+\hat{s} \xi(\phi))^{-1}\left[1+R_{, i}^{i}(\phi) \xi(\phi)\right]
\end{aligned}
$$

and arrive at the following presentation of the generating functional $Z(J, K)$

$$
\begin{array}{r}
Z(J, K)=\int \mathscr{D} \phi \exp \left\{\frac{i}{\hbar}[S(\phi, K)+J(\phi+R(\phi) \xi(\phi))-\right. \\
\left.\left.-i \hbar R_{, i}^{i}(\phi) \xi(\phi)+i \hbar \ln (1+\hat{s} \xi(\phi))\right]\right\} .
\end{array}
$$

Due to the equivalence theorem [12] we can work with the following generating functional

$$
Z_{\xi}(J, K)=\int \mathscr{D} \phi \exp \left\{\frac{i}{\hbar}\left[S_{\xi}(\phi, K)+J \phi\right]\right\}
$$

where

$$
S_{\xi}(\phi, K)=S(\phi, K)+i \hbar \ln (1+\hat{s} \xi(\phi))-i \hbar R_{, i}^{i}(\phi) \xi(\phi) .
$$


In its turn, in general, the action $S_{\xi}(\phi, K)$ is not invariant under fermion transformations

$$
\hat{s} S_{\xi}(\phi, K)=-i \hbar \hat{s}\left(R_{, i}^{i}(\phi) \xi(\phi)\right) \neq 0 .
$$

We consider this as an indication of the inconsistency in formulation of the model being invariant under fermion transformations. Indeed, it seems strange that a theory with the action invariant under fermion transformations is equivalently presented in the form when this symmetry looks like broken. This inconsistency can be avoided if the additional requirement is fulfilled, $R_{, i}^{i}(\phi)=0$.

\section{Special fermion transformations}

We will refer special type of theories when generators $R^{i}(\phi)$ of fermion symmetry transformations are subjected to the restriction

$$
R_{, i}^{i}(\phi)=0
$$

In the case of such theories the superdeterminant of field-dependent fermion transformations reads

$$
\mathrm{sDet} M=(1+\hat{s} \xi)^{-1}
$$

and for the action $S_{\xi}$ one has

$$
S_{\xi}(\phi, K)=S(\phi, K)+i \hbar \ln (1+\hat{s} \xi(\phi)) .
$$

There is the presentation

$$
S_{\xi}(\phi, K)=S(\phi, K)+\hat{s} F(\phi)=S(\phi)+\hat{s}(K \phi+F(\phi))
$$

where

$$
F=\xi\left[1-\frac{1}{2}(\hat{s} \xi)+\frac{1}{3}(\hat{s} \xi)^{2}-\cdots\right]=\xi(\hat{s} \xi)^{-1} \ln (1+\hat{s} \xi) .
$$

This presentation can be very useful in theories with the action invariant under fermion transformation. In particular, it was shown [10] that for Yang-Mills theories the result of change of variables can be presented in the similar form and interpreted as a modification of the gauge condition. This made it possible to prove the independence of the effective action in Yang-Mills theories on the finite increment of gauge on-shell and suggest the formulation of the Gribov-Zwanziger theory $[13,14]$ free from the problem of gauge dependence $[10,15]$.

The action $S_{\xi}(\phi, K)$ is invariant under fermion transformations

$$
\hat{s} S_{\xi}(\phi, K)=0
$$

This invariance can be expressed in the form of Zinn-Justin equation

$$
\frac{\delta S_{\xi}}{\delta \phi^{i}} \frac{\delta S_{\xi}}{\delta K^{i}}=0
$$


As a consequence the generating functional $Z_{\xi}(J, K)$ satisfies the Ward identity

$$
J_{i} \frac{\delta Z_{\xi}(J, K)}{\delta K_{i}}=0
$$

like $Z(J, K)$. One can rewrite the Ward identity in term of the generating functional of connected Green functions $W_{\xi}(J, K)=(\hbar / i) \ln Z_{\xi}(J, K)$ as

$$
J_{i} \frac{\delta W_{\xi}(J, K)}{\delta K_{i}}=0 .
$$

Using the Legendre transformation

$$
\phi^{i}=\frac{\delta W_{\xi}(J, K)}{\delta J_{i}}
$$

and introducing the generating functional of vertex functions $\Gamma_{\xi}(\phi, K)$

$$
\Gamma_{\xi}(\phi, K)=W_{\xi}(J, K)-J_{i} \phi^{i}, \quad \frac{\delta \Gamma_{\xi}}{\delta K_{i}}=\frac{\delta W_{\xi}}{\delta K_{i}}, \quad \frac{\delta \Gamma_{\xi}}{\delta \phi^{i}}=-J_{i},
$$

the Ward identity for $\Gamma_{\xi}=\Gamma_{\xi}(\phi, K)$

$$
\frac{\delta \Gamma_{\xi}}{\delta \phi^{i}} \frac{\delta \Gamma_{\xi}}{\delta K_{i}}=0
$$

has the form of the Zinn-Justin equation and repeats on quantum level the invariance of the given theory under fermion transformations. It is clear that all relations are valid for the initial theory $(\xi=0)$.

\section{Conclusions}

It seems interesting from general point of view to study quantum properties of field theories for which an action appearing in the generating functional of Green functions is invariant under fermion transformations.

In turn the fermion transformations can be of three types. The first type is characterized as fermion transformations when there are no any restrictions on generators of these transformations. The second type consists of nilpotent fermion transformations. The third type is characterized as nilpotent fermion transformations subjected to an additional restriction on generators.

\section{References}

[1] L.D. Faddeev and V.N. Popov, Feynman diagrams for the Yang-Mills field, Phys. Lett. B25 (1967) 29.

[2] C. Becchi, A. Rouet and R. Stora, Renormalization of the abelian Higgs-Kibble model, Commun. Math. Phys. 42 (1975) 127.

[3] I.V. Tyutin, Gauge invariance in field theory and statistical physics in operator formalism, Lebedev Inst. preprint N 39 (1975), arXiv:0812.0580 [hep-th] .

[4] C. Curci, R. Ferrari, Slavnov transformations and supersymmetry, Phys. Lett. B63 (1976) 91. 
[5] E.S. Fradkin and G.A. Vilkovisky, Quantization of relativistic systems with constraints, Phys. Lett. B55 (1975) 224.

[6] I.A. Batalin and G.A. Vilkovisky, Relativistic S matrix od dinamical systems with boson and fermion constraints, Phys. Lett. B69 (1977) 309.

[7] S. Catterall and S. Chadab, Lattice sigma models with exact supersymmetry, JHEP 0405 (2004) 044.

[8] A. Quagri and A.A. Slavnov, Renormalization of the Yang-Mills theory in the ambiguity-free gauge, JHEP 1007 (2010) 087.

[9] A. Jourjine, Scalar Supersymmetry and Fermiophobic Higgs, Phys. Lett. B727 (2013) 211.

[10] P.M. Lavrov and O. Lechtenfeld, Field-dependent BRST transformations in Yang-Mills theory, Phys.Lett. B725 (2013) 382.

[11] J. Zinn-Justin, Renormalization of gauge theories, in Trends in Elementary Particle Theory, Lecture Notes in Physics, Vol. 37, ed. H.Rollnik and K.Dietz (Springer-Verlag, Berlin, 1975).

[12] R.E. Kallosh, I.V. Tyutin, The equivalence theorem and gauge invariance in renormalizable theories, Sov. J. Nucl. Phys. 17 (1973) 190.

[13] V.N. Gribov, Quantization of nonabelian gauge theories, Nucl. Phys. B139 (1978) 1.

[14] D. Zwanziger, Action from the Gribov horizon, Nucl. Phys. B321 (1989) 591.

[15] P. Lavrov, O. Lechtenfeld and A. Reshetnyak, Is soft breaking of BRST symmetry consistent?, JHEP 1110 (2011) 043. 